October 1997

TMUP-HEL-9710

UM-P-97/57

RCHEP-97/14

\title{
Up-down atmospheric neutrino flux asymmetry predictions for various neutrino oscillation scenarios
}

\author{
R. Foot ${ }^{a}$, R. R. Volkas ${ }^{a}$ and O. Yasuda ${ }^{b}$ \\ a School of Physics, \\ Research Centre for High Energy Physics, \\ The University of Melbourne, \\ Parkville, 3052 Australia. \\ ${ }^{b}$ Department of Physics, Tokyo Metropolitan University, \\ 1-1 Minami-Osawa Hachioji, Tokyo 192-03, Japan
}

\begin{abstract}
We compute up-down asymmetries for atmospheric neutrino induced electron and muon events in the SuperKamiokande detector for the following neutrino oscillation models: (A) maximally mixed $\nu_{\mu}-\nu_{\tau}$ or $\nu_{\mu}-\nu_{s}$, (B) maximally mixed $\nu_{\mu}-\nu_{e},(\mathrm{C})$ threefold maximal mixing between $\nu_{e}, \nu_{\mu}$ and $\nu_{\tau}$, and (D) neutrino oscillations via Equivalence Principle or Lorentz invariance violation. We emphasise the role of different momentum cuts.
\end{abstract}


The solar [1] and atmospheric [2]-[7] neutrino observations and the LSND [8] experiment provide strong evidence that neutrinos have nonzero masses and oscillate. Many specific neutrino mass scenarios have been proposed as explanations for some or all of these results. These explanations will be put to stringent tests in the next few years as more data are collected.

In this paper we will focus on ways to experimentally test several different proposed explanations of the atmospheric neutrino anomaly. One good way to discriminate between these possible explanations is through the up-down asymmetry, with respect to zenith angle, of the detected charged leptons in experiments such as SuperKamiokande [9] 10] 11. This idea was introduced in Ref.[9] and studied in detail in Ref. [10] in the context of the Exact Parity Model solution to the atmospheric neutrino anomaly [12]. The updown asymmetry idea was independently considered by the authors of Ref. [11] in the case of six possible neutrino oscillation models. As emphasised in Ref. [11], the sign and energy dependence of the up-down asymmetry will provide a useful diagnostic tool in differentiating the various explanations of the atmospheric neutrino anomaly. Reference 111 focussed on the energy dependence of the up-down asymmetry. A complementary approach is to study the asymmetry with various cuts in momentum [10] [13]. This is useful because the Kamiokande and SuperKamiokande collaborations have divided their event sample into two classes (sub-GeV and multi-GeV) on the basis of momentum cuts. In the future, improved statistics may lead to the event sample being further subdivided with respect to momentum. The purpose of this paper is to study the up-down asymmetry expected at SuperKamiokande with various cuts in momentum for a variety of neutrino oscillation models.

It is a priori interesting to consider various cuts in zenith angle as well as energy when analysing the up-down asymmetries of charged lepton events in SuperKamiokande [10]. A class of measures of the up-down asymmetry can be defined by introducing the quantities $y_{e}^{\eta}$ and $y_{\mu}^{\eta}$, where

$$
y_{e}^{\eta} \equiv \frac{N_{e}^{-\eta}}{N_{e}^{+\eta}}, \quad y_{\mu}^{\eta} \equiv \frac{N_{\mu}^{-\eta}}{N_{\mu}^{+\eta}} .
$$

Here $N_{e}^{-\eta}\left(N_{e}^{+\eta}\right)$ is the number of charged electrons produced in the detector with zenith angle $\cos \Theta<-\eta(\cos \Theta>\eta)$. $\quad N_{\mu}^{ \pm \eta}$ are the analogous quantities for charged muons. The zenith angle $\Theta$ is defined so that $\Theta=0$ corresponds to downward travelling and $\Theta=\pi$ to upward travelling charged leptons. Different choices for $\eta$ correspond to different cuts in zenith angle. (Note that Ref.[11] considered the $\eta=0$ case in detail for several different models, while Ref. [10] studied different choices for $\eta$ for the Exact Parity Model.) Since $y_{e, \mu}^{\eta}$ are ratios of fluxes, they will be approximately free of the systematic errors arising from uncertain absolute fluxes $( \pm 30 \%)$ as well as uncertain $\mu / e$ flux ratios $( \pm$ a few percent). For symmetry reasons, $y_{e, \mu}^{\eta} \simeq 1$ is expected in the absence of neutrino oscillations. If neutrino oscillations are present, however, then $y_{e, \mu}^{\eta} \neq 1$ is expected. 
In the calculations to be presented later, we actually use the related quantities $Y_{e, \mu}^{\eta}$, where

$$
Y_{e, \mu}^{\eta} \equiv \frac{\left.y_{e, \mu}^{\eta}\right|_{o s c}}{\left.y_{e, \mu}^{\eta}\right|_{n o-o s c}} .
$$

The numerators are the predictions for $y_{e, \mu}^{\eta}$ in the models, while the denominators are the same quantities in the absence of oscillations. We will focus on the case

$$
\eta=0.2
$$

because our experience with various values for $\eta$ in Ref. [10] suggests that this choice leads to the largest effects without compromising too much in regard to statistics. Using $\eta=0.2$ also allows comparison of our results with the preliminary data from SuperKamiokande.

The cases we consider are

(A) Maximal $\nu_{\mu}-\nu_{\tau}$ [14] or $\nu_{\mu}-\nu_{s}$ mixing [12]. These two scenarios are indistinguishable as far as up-down asymmetries are concerned provided that matter effects can be neglected (which is approximately the case for the region of $\delta m^{2}$ considered in this paper). The $\nu_{\mu}-\nu_{s}$ scenario is, in particular, motivated by the Exact Parity Model in the region of parameter space where $\nu_{e}-\nu_{s}^{\prime}$ oscillations can be neglected for atmospheric neutrinos (Ref. [10] focussed on the parameter space region where $\nu_{e}$ oscillations are also important). (B) Maximal $\nu_{\mu}-\nu_{e}$ mixing. The Acker-Pakvasa three-flavour model [16] is essentially indistinguishable from this scenario for atmospheric neutrinos.

(C) Threefold maximal mixing [17] [18] amongst $\nu_{e}, \nu_{\mu}$ and $\nu_{\tau}$ in the region of parameter space considered in Ref. [18].

(D) Massless neutrinos with violation of the Equivalence Principle or breakdown of Lorentz invariance [19]. The special case of exactly maximal $\nu_{e}-\nu_{\mu}$ oscillations[20] is considered for definiteness $\left(\sin ^{2} 2 \theta\right.$ can actually be as low as 0.8 phenomenologically).

The Cardall-Fuller three-flavour scheme [15] is numerically similar to case A with the parameter choice $\delta m^{2} \simeq 0.3 \mathrm{eV}^{2}$. For such a large $\delta m^{2}$ there are no expected up-down asymmetries for the sub-GeV or multi-GeV SuperKamiokande data.

Consider sub-GeV neutrinos first. In the water-Cerenkov Kamiokande and SuperKamiokande experiments, the sub- $\mathrm{GeV}$ neutrinos are detected via the charged leptons $\ell_{\alpha}$ $\left(\ell_{\alpha}=e\right.$ or $\mu$ ) produced primarily from the quasi-elastic neutrino scattering off nucleons in the water molecules: $\nu_{\alpha} N \rightarrow \ell_{\alpha} N^{\prime}(\alpha=e, \mu)$. The event rate is calculated by integrating the product of the differential neutrino flux, the scattering differential cross-section, the energy efficiency function for the detector and the relevant neutrino oscillation probability with respect to energy and angular variables (see, for example, Ref. [10] for more details). In our numerical work we have used the differential cross section in Ref.[21]. The differential flux of atmospheric neutrinos without geomagnetic effects is given in [22], but we have used the differential flux which includes geomagnetic effects [23]. (For other atmospheric 
neutrino flux calculations, see Ref.[24].) The detector energy efficiency function can be found in Ref. 225].

In principle, the charged lepton event rates for multi-GeV neutrinos require a more involved computation, because several scattering channels must be considered. Fortunately, as noted in Ref. [11], the up-down asymmetries are generally insensitive to these details. In practice, all one really needs to calculate up-down asymmetries reliably is a reasonable estimate for a function which is effectively a product of the cross-section and the energy efficiency function. We have estimated this function from the SuperKamiokande MonteCarlo calculations of event rates in the absence of oscillations. We numerically checked the dependence of the asymmetries on variations in this "effective cross-section". Cases $\mathrm{A}, \mathrm{B}$ and $\mathrm{C}$ vary by only a few percent, while case $\mathrm{D}$ turns out to be uncertain to about the $10 \%$ level.

In Figures 1-6 we plot $Y_{e, \mu}^{0.2}$ for each of the cases $\mathrm{A}, \mathrm{B}, \mathrm{C}$ and $\mathrm{D}$, for three different momentum cuts, as a function of the relevant parameter [26]. For case A the parameter is the difference of squared masses between $\nu_{\mu}$ and either $\nu_{\tau}$ or $\nu_{s}$. For case B it is the $\delta m^{2}$ between $\nu_{\mu}$ and $\nu_{e}$. In case $\mathrm{C}$ it is the larger of the two independent $\delta m^{2}$ 's, while for case $\mathrm{D}$ it is the usual measure of either Equivalence Principle or Lorentz invariance violation. In Figs. 1 and 2 we have utilised the standard Kamiokande sub-GeV momentum cuts, $0.2<$ $p_{\mu} / G e V<1.5$ and $0.1<p_{e} / G e V<1.33$, for muons and electrons respectively. Figures 3 and 4 show the corresponding plots when the alternative cuts, $0.5<p_{\mu} / G e V<1.5$ and $0.5<p_{e} / \mathrm{GeV}<1.33$, are employed. Figures 5 and 6 pertain to multi-GeV events. We now discuss some of the important qualitative features of the graphs:

Most of these curves show that the asymmetry depends weakly on the neutrino oscillation parameter $\left(\delta m^{2}\right.$ or $\left.\delta v / 2\right)$ over a significant range (almost an order of magnitude). This can be understood as follows: Neutrinos with $\cos \theta>0.2$ travel distances $15 \lesssim \mathrm{L} / \mathrm{km} \lesssim$ 75 , whereas neutrinos with $\cos \theta<-0.2$ travel distances $2600 \lesssim L / k m \lesssim 12,700$. Thus there will be a large range of $\delta m^{2}$ or $\delta v / 2$ where the oscillation length is such that neutrinos with $\cos \theta>0.2$ do not have time to oscillate, whereas neutrinos with $\cos \theta<-0.2$ oscillate and are averaged. This weak dependence is interesting because it effectively provides a prediction within each model for the asymmetries which is reasonably insensitive to parameter choice and hence avoids the potential problem of fine-tuning. (Note that the plateau feature in the asymmetries is also there when $\eta=0$ is used instead of $\eta=0.2$. The point is that, due to the geometry of the situation, the neutrino flux from the intermediate regime $-0.2<\cos \Theta<0.2$ forms a small enough fraction of the total flux that the qualitative plateau phenomenon persists.)

A comparison of Figs. 1 and 3 shows that case A can be more clearly distinguished from the other cases by adopting the alternative sub-GeV momentum cut. Case A is in turn clearly different from the no-oscillation $Y=1$ case. Cases B and D can be distinguished from case $\mathrm{C}$ through electron asymmetries, particularly using the alternative sub-GeV cut, according to Figs. 2 and 4. Although case $\mathrm{C}$ is not clearly different from the no- 
oscillation situation for electron asymmetries, one can return to the muon asymmetries of Figs. 1 and 2 to obtain a clear differentiation. The most problematic differentiation for the sub-GeV sample is evidently that between cases B and D. If $\delta m^{2}$ or $\delta v / 2$ is sufficently large $\left[>\sim 10^{-2} \mathrm{eV}^{2}\right.$ or $\left.(\mathrm{km} \mathrm{GeV})^{-1}\right]$, then this discrimination can be more easily made. Fortunately, the multi-GeV cases displayed in Figs. 5 and 6 show a clear difference between cases $\mathrm{B}$ and $\mathrm{D}$ unless $\delta m^{2}$ or $\delta v / 2$ is quite small.

Preliminary atmospheric neutrino results from SuperKamiokande can be found in Ref.[27]. The asymmetries $Y_{e, \mu}^{0.2}$ can be estimated from the graphs presented for the sub$\mathrm{GeV}$ case with the usual momentum cut and for the multi-GeV case. The results are,

$$
Y_{e}^{0.2}(s u b-G e V) \simeq 1.19 \pm 0.10(\text { stat. }) \quad Y_{\mu}^{0.2}(s u b-G e V) \simeq 0.80 \pm 0.07 \text { (stat.) }
$$

and

$$
\left.Y_{e}^{0.2}(\text { multi }-G e V) \simeq 0.80 \pm 0.15 \text { (stat. }\right) \quad Y_{\mu}^{0.2}(\text { multi }-G e V) \simeq 0.46 \pm 0.07 \text { (stat.) }
$$

These numbers can be compared to Figures 1, 2, 5 and 6. These figures show that while case A fits the preliminary data best, the still significant statistical errors preclude definitive conclusions. The Cardall-Fuller model fits the data least well.

In summary, up-down asymmetries in the charged leptons induced by atmospheric neutrinos in the SuperKamiokande detector are important quantities that, with improved statistics, will be capable of clearly distinguishing the various proposed solutions to the atmospheric neutrino anomaly.

\section{Acknowledgements}

This work was supported in part by the Australian Research Council. R.F. is an Australian Research Fellow.

\section{References}

[1] Homestake Collab., Nucl. Phys. B. Proc. Suppl. 38, 47 (1995); Kamiokande Collab., Nucl. Phys. B. Proc. Suppl. 38, 55 (1995); Gallex Collab., Phys. Lett. B327, 377 (1994); Sage Collab., Phys. Lett. B328, 234 (1994).

[2] Kamiokande Collaboration, K. S. Hirata et al., Phys. Lett. B205, 416 (1988); ibid. B280, 146 (1992).

[3] Kamiokande Collaboration, Y. Fukuda et al., Phys. Lett. B335, 237 (1994). 
[4] IMB Collaboration, D. Casper et al., Phys. Rev. Lett. 66, 2561 (1989); R. BeckerSzendy et al., Phys. Rev D46, 3720 (1989).

[5] NUSEX Collaboration, M. Aglietta et al., Europhys. Lett. 8, 611 (1989).

[6] Frejus Collaboration, Ch. Berger et al., Phys. Lett. B227, 489 (1989); ibit. B245, 305 (1990); K. Daum et al, Z. Phys. C66 417 (1995).

[7] Soudan 2 Collaboration, M. Goodman et al., Nucl. Phys. B (Proc. Suppl.) 38,337 (1995); W.W.M., Allison et. al., Phys. Lett. B391, 491 (1997).

[8] LSND Collab., C. Athanassopoulos et. al., Phys. Rev. Lett. 75, 2650 (1995); Phys. Rev. C54, 2685 (1996); and preprint UCRHEP-E-191, nucl-ex/9706006 (1997).

[9] J. Bunn, R. Foot and R. R. Volkas, hep-ph/9702429 (Phys. Lett. B, in press).

[10] R. Foot, R. R. Volkas and O. Yasuda, preprint TMUP-HEL-97/07, hep-ph/9709483.

[11] J. W. Flanagan, J. G. Learned and S. Pakvasa, preprint UH511-880-97, hep$\mathrm{ph} / 9709438$.

[12] R. Foot and R. R. Volkas, Phys. Rev. D52, 6595 (1995) and references therein.

[13] Note that energy and momentum are almost interchangeable quantities except for muon events in the 100's of MeV range.

[14] J. G. Learned, S. Pakvasa and T. J. Weiler, Phys. Lett. B207, 79 (1988); V. Barger and K. Whisnant, Phys. Lett. B209, 365 (1988); K. Hidaka, M. Honda and S. Midorikawa, Phys. Rev. Lett. 61, 1537 (1988).

[15] C. Y. Cardall and G. Fuller, Phys. Rev. D53, 4421 (1996).

[16] A. Acker and S. Pakvasa, Phys. Lett. B397, 209 (1997); A. Acker, J. G. Learned, S. Pakvasa and T. Weiler, Phys. Lett. B298, 149 (1993).

[17] C. Giunti, C. W. Kim and J. D. Kim, Phys. Lett. B352, 357 (1995). See also R. N. Mohapatra and S. Nussinov, Phys. Lett. B346, 75 (1995).

[18] P. F. Harrison, D. H. Perkins and W. G. Scott, Phys. Lett. B396, 186 (1997); Phys. Lett. B349, 137 (1995).

[19] M. Gasperini, Phys. Rev. D38, 2635 (1988); A. Halprin and C. N. Leung, Phys. Rev. Lett. 67, 1833 (1991); K. Iida, H. Minakata and O. Yasuda, Mod. Phys. Lett. A8, 1037 (1993); S. Coleman and S. L. Glashow, preprint HUTP-97/A008, hepph/9703240; S. L. Glashow et al., Phys. Rev. D56, 2433 (1997); J. Pantaleone, A. 
Halprin and C. N. Leung, Phys. Rev. D47, 4199 (1993); J. R. Mureika and R. B. Mann, Phys. Rev. D54, 2761 (1996).

[20] In this case the survival probability is given by $P_{e e}=P_{\mu \mu}=1-\sin ^{2} \frac{\delta v}{2} E L$ and the transition probability is $P_{e \mu}=P_{\mu e}=\sin ^{2} \frac{\delta v}{2} E L$ (where $E$ and $L$ are the energy of the neutrino and the distance travelled respectively).

[21] T. K. Gaisser and J. S. O'Connell, Phys. Rev. D34, 822 (1986).

[22] M. Honda, T. Kajita, S. Midorikawa, and K. Kasahara, Phys. Rev. D52, 4985 (1995).

[23] M. Honda, K. Kasahara, and S. Midorikawa, private communication.

[24] L.V. Volkova, Sov. J. Nucl. Phys. 31, 784 (1980); T.K. Gaisser, T. Stanev S.A. Bludman and H. Lee, Phys. Rev. Lett. 51, 223 (1983); A. Dar, Phys. Rev. Lett. 51, 227 (1983); K. Mitsui, Y. Minorikawa and H. Komori, Nuovo Cim. C9, 995 (1986); E.V. Bugaev and V.A. Naumov, Sov. J. Nucl. Phys. 45, 857 (1987); T.K. Gaisser, T. Stanev and G. Bar, Phys. Rev. D38, 85 (1988); A.V. Butkevich, L.G. Dedenko and I.M. Zheleznykh, Sov. J. Nucl. Phys. 50, 90 (1989); M. Honda, K. Kasahara, K. Hidaka and S. Midorikawa, Phys. Lett. B248, 193 (1990); H. Lee and Y. S. Koh, Nuovo Cim. B105, 883 (1990); M. Kawasaki and S. Mizuta, Phys. Rev. D43, 2900 (1991); P. Lipari, Astropart. Phys. 1, 195 (1993). D.H. Perkins, Astropart. Phys. 2, 249 (1994); V. Agrawal, T.K. Gaisser, P. Lipari and T. Stanev, Phys. Rev. D53, 1314 (1996); T.K. Gaisser and T. Stanev, astro-ph/9708146.

[25] T. Kajita, Physics and Astrophysics of Neutrinos eds. M. Fukugita and A. Suzuki (Springer-Verlag, Tokyo, 1994), p559.

[26] In our numerical work we found some small oscillations of the $Y$ parameter which we have smoothed out in the Figures (the oscillations are typically less than 5 percent).

[27] M. Nakahata, Talk at International Europhysics Conference on High Energy Physics, August 19-26, Jerusalem, Israel; Y. Totsuka, Talk at XVIII International Symposium on Lepton-Photon Interactions, July 28 - August 1, Hamburg, Germany (http://www.desy.de/lp97-docs/talks/P22.ps). 


\section{Figure Captions}

Figure 1. $Y_{\mu}^{0.2}$ with momentum cut $0.2<p_{\mu} / G e V<1.5$ for case A (solid line), B (dasheddotted line), $\mathrm{C}$ (dashed line) and $\mathrm{D}$ (dotted line). Note $x=\delta m^{2}\left(\mathrm{eV}^{2}\right)$ for cases A, B and $\mathrm{C}$ while $x=\delta v / 2\left(\mathrm{~km}^{-1} \mathrm{GeV}^{-1}\right)$ for case D. Also shown (straight dotted lines) is the preliminary superKamiokande result (Eq.(蛋) together with 1 sigma statistical errors.

Figure 2. $Y_{e}^{0.2}$ with momentum cut $0.1<p_{e} / G e V<1.33$ for the cases A (solid line), B (dashed-dotted line), C (dashed line) and D (dotted line). Also shown (straight dotted lines) is the preliminary superKamiokande result (Eq.(佃) together with 1 sigma statistical errors.

Figure 3. Same as Figure 1, except that the momentum cut $0.5<p_{\mu} / G e V<1.5$ has been taken.

Figure 4. Same as Figure 2, except that the momentum cut $0.5<p_{e} / G e V<1.33$ has been taken.

Figure 5. $Y_{\mu}^{0.2}$ for the SuperKamiokande multi-GeV sample. Also shown (straight dotted lines) is the preliminary superKamiokande result (Eq.(5) together with 1 sigma statistical errors.

Figure 6. $Y_{e}^{0.2}$ for the SuperKamiokande multi-GeV sample. Also shown (straight dotted lines) is the preliminary superKamiokande result (Eq.(5) together with 1 sigma statistical errors. 


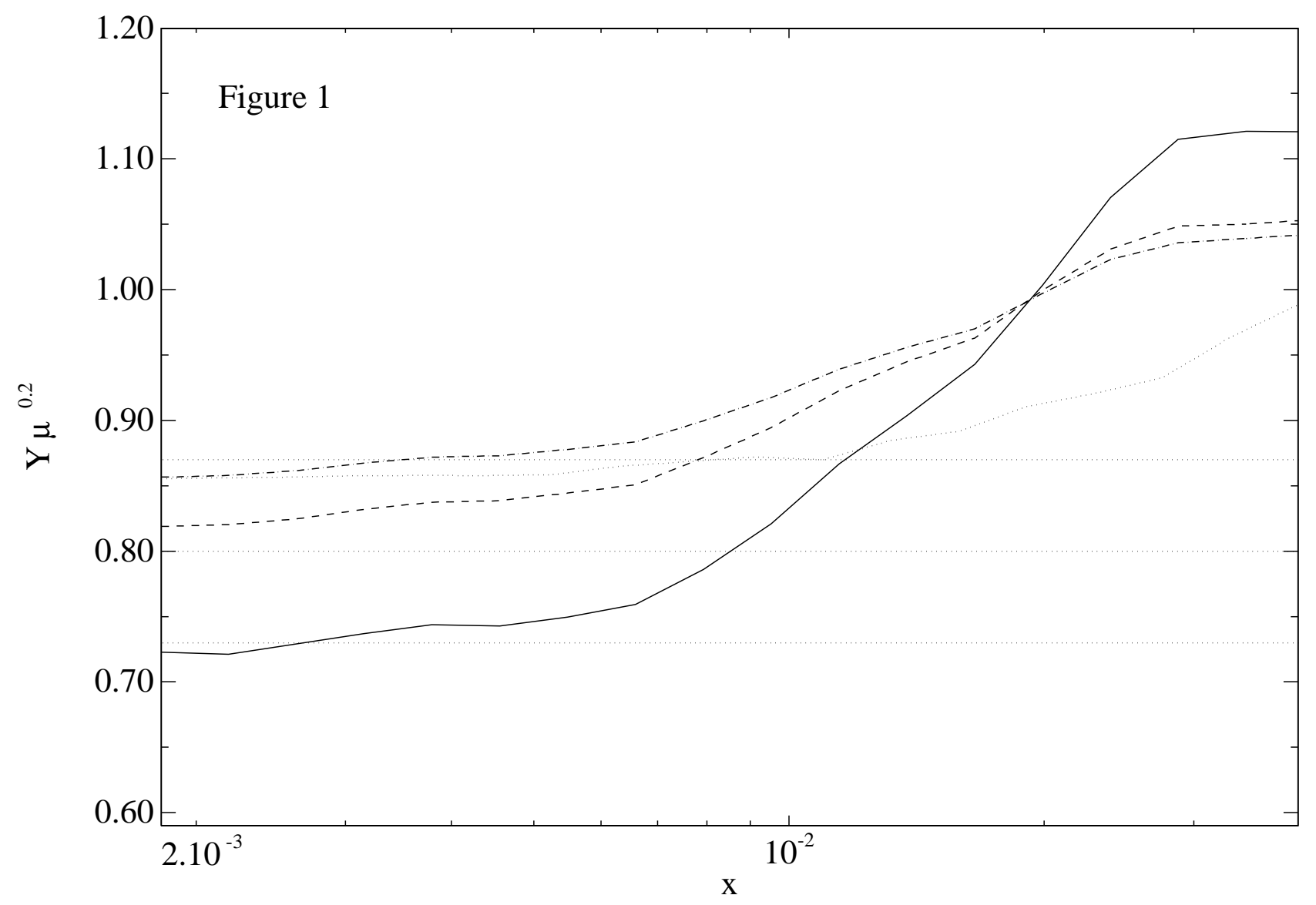




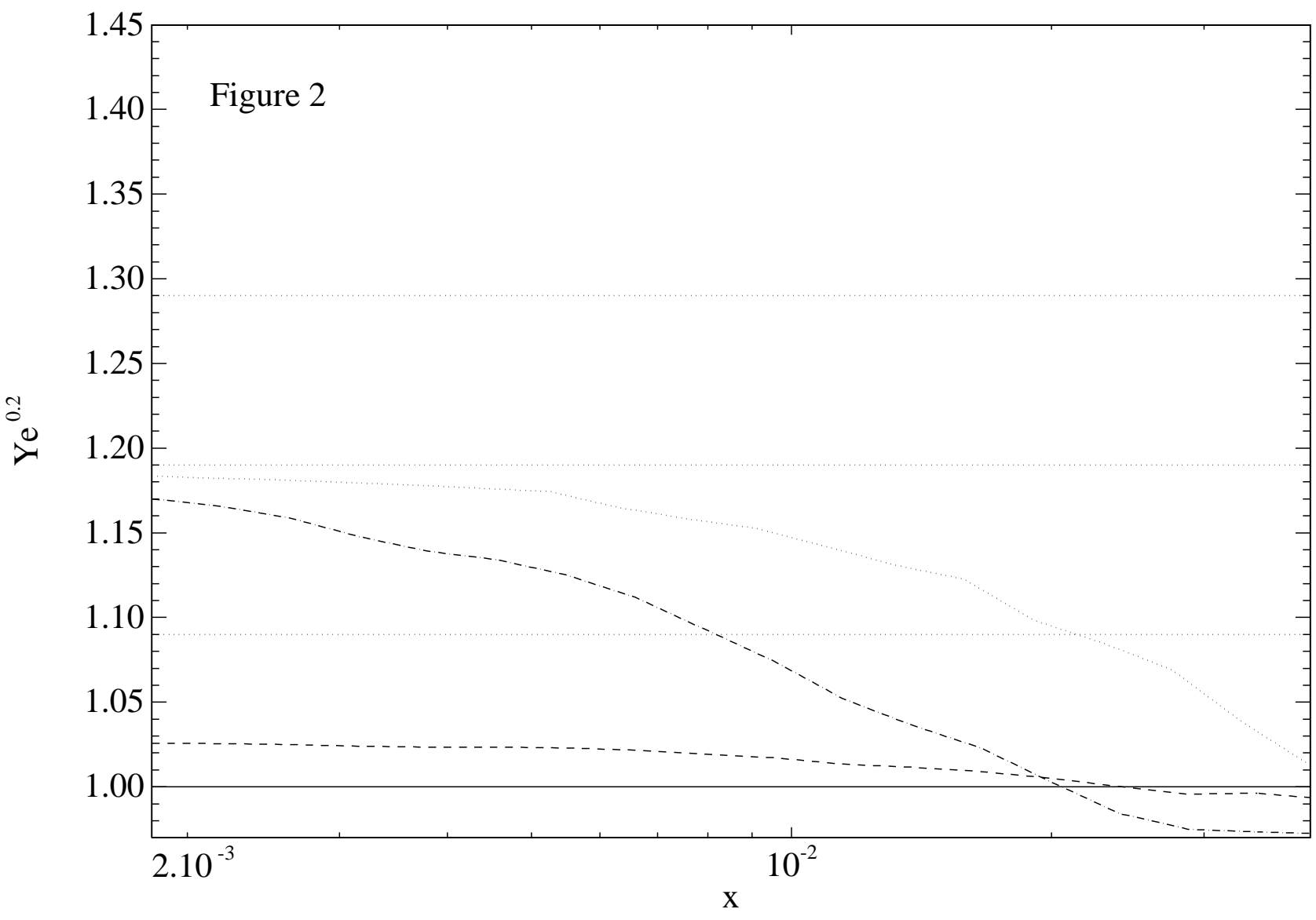




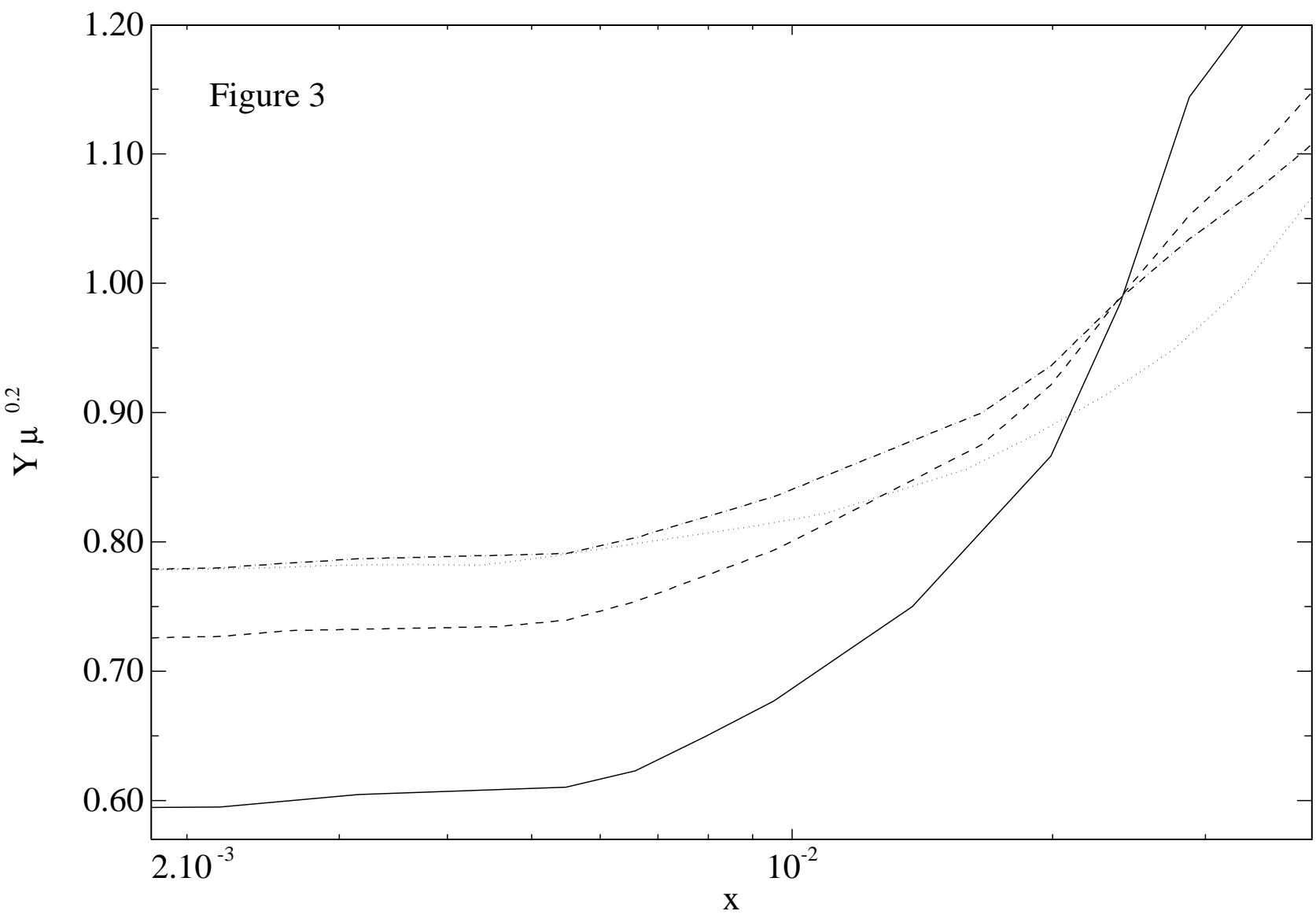




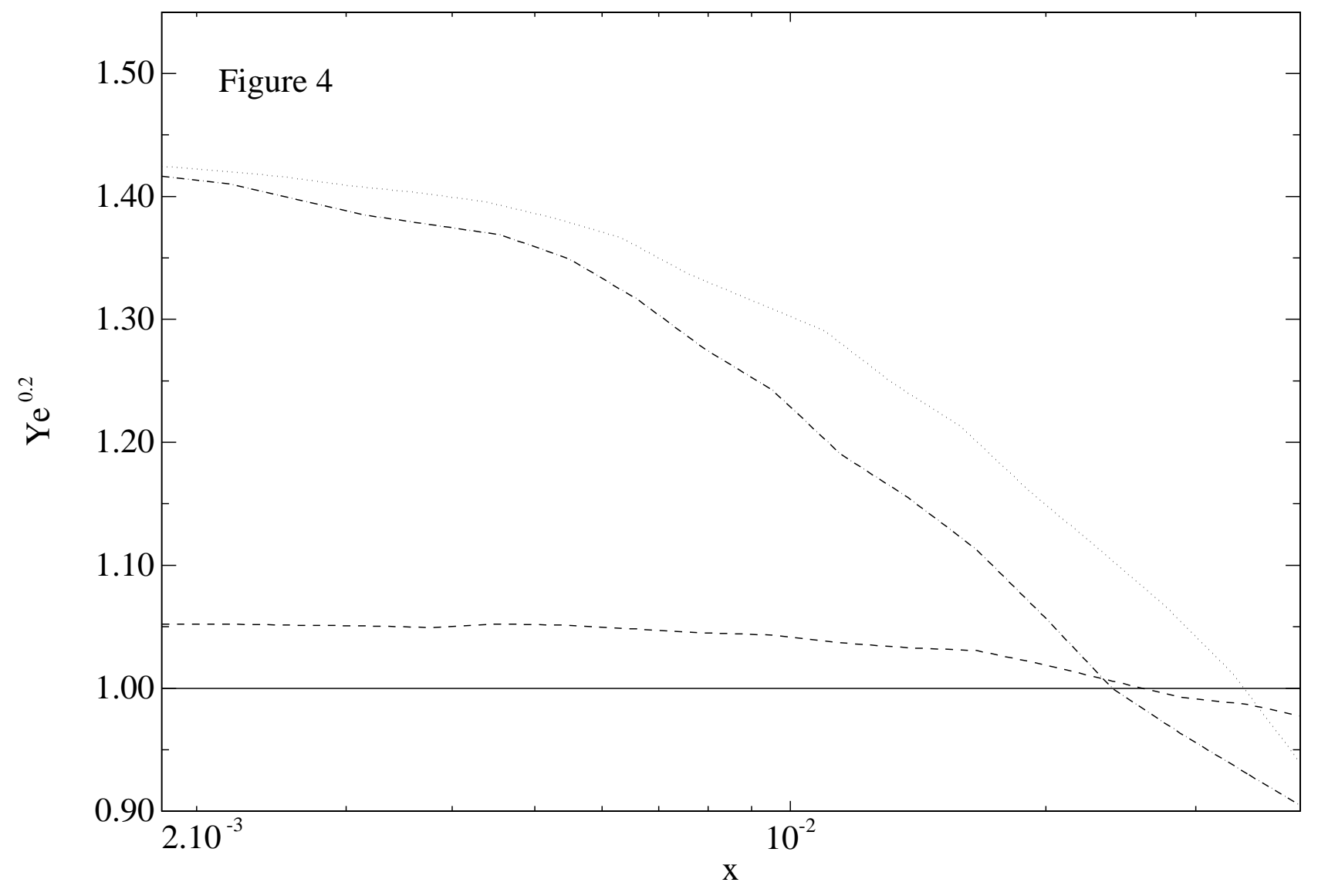




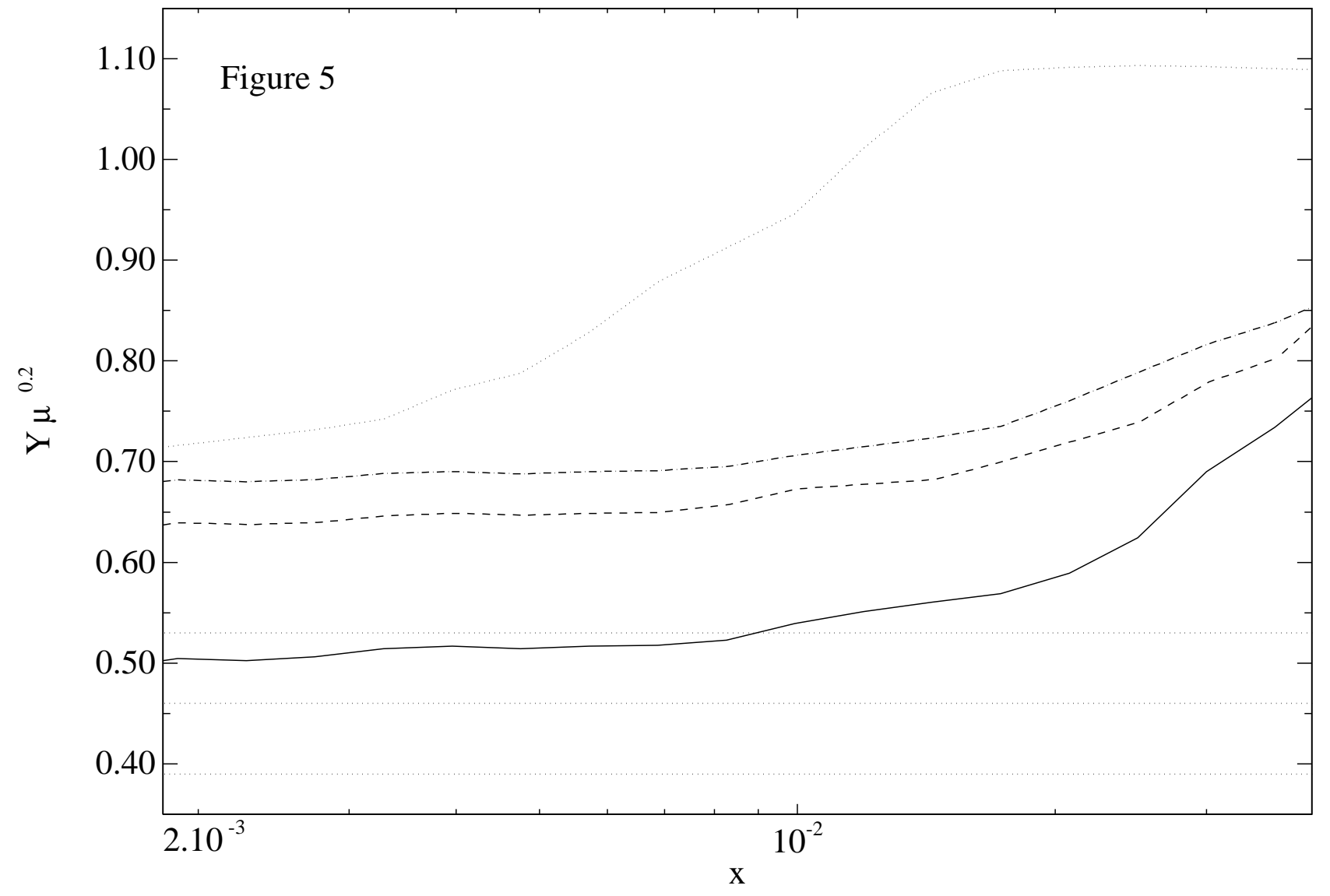




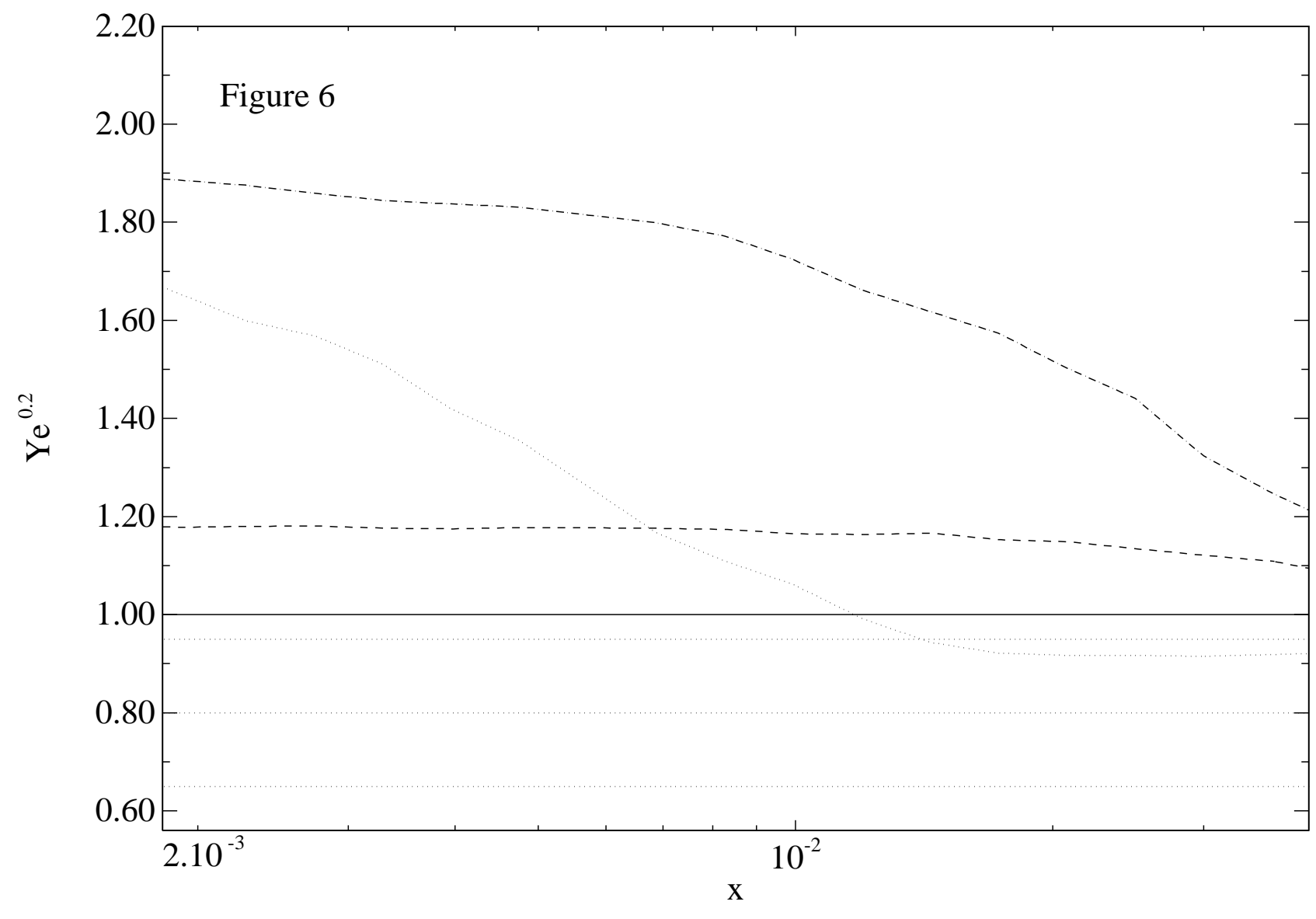

